\title{
Association between maternal sleep duration and quality, and the risk of preterm birth: a systematic review and meta-analysis of observational studies
}

\author{
Ling Wang and Feng Jin
}

\begin{abstract}
Background: To assess the association of sleep duration and quality with the risk of preterm birth.

Methods: Relevant studies were retrieved from the PubMed and Web of Science databases up to September 30, 2018. The reference lists of the retrieved articles were reviewed. Random effects models were applied to estimate summarized relative risks (RRs) and 95\% confidence intervals (Cls).

Results: Ten identified studies (nine cohort studies and one case-controlled study) examined the associations of sleep duration and quality with the risk of preterm birth. As compared with women with the longest sleep duration, the summary RR was $1.23(95 \% \mathrm{Cl}=1.01-1.50)$ for women with the shortest sleep duration, with moderate between-study heterogeneity $\left(P^{2}=57.4 \%\right)$. Additionally, as compared with women with good sleep quality, the summary RR was $1.54(95 \% \mathrm{Cl}=1.18-2.01)$ for women with poor sleep quality (Pittsburgh Sleep Quality Index > 5), with high between-study heterogeneity $\left(I^{2}=76.7 \%\right)$. Funnel plots as well as the Egger's and Begg's tests revealed no evidence of publication bias.

Conclusions: This systematic review and meta-analysis revealed that short sleep duration and poor sleep quality may be associated with an increased risk of preterm birth. Further subgroup analyses are warranted to test the robustness of these findings as well as to identify potential sources of heterogeneity.
\end{abstract}

Keywords: Meta-analysis, Observational studies, Preterm birth, Sleep, Systematic review

\section{Background}

Each year, more than $10 \%$ of births worldwide are preterm, occurring before gestational week 37. Prematurity, which is a leading cause of mortality among children aged less than 5 years [1, 2], leads to short and longterm morbidities, as well as serious health problems, including both physical and mental disabilities [3-5]. Recently, increasing numbers of studies have sought to identify risk factors associated with preterm birth. Moreover, a recent review summarized the risk factors for prematurity, which included pregnancy-related depression, stress, and anxiety [6], while preexisting diabetes,

\footnotetext{
* Correspondence: jinf13520@163.com

Department of Obstetrics and Gynecology, Shengjing Hospital of China Medical University, No. 36, San Hao Street, Shenyang, Liaoning 110004,

People's Republic of China
}

hypertension, sickle cell anemia, and previous preterm birth were associated with a two-fold greater risk of preterm birth [7].

Due to psychophysiological changes caused by pregnancy, approximately $27.9 \%$ of women sleep for less than $7 \mathrm{~h}$ per night because of sleep disturbances [8]. Thus, numerous studies have investigated the relationship between maternal sleep practices and fetal outcomes. For instance, insomnia and sleep apnea in pregnancy increased the risk of preterm birth by 30 and $40 \%$, respectively [9]. Moreover, pregnancies complicated by restless legs syndrome are also at an increased risk for preterm birth [10], while pregnancy-related endocrinological and physical changes can also result in sleep disturbances [11]. As compared with the general population, pregnant women are more likely to encounter sleep-related 
disorders, including poor subjective sleep quality and shorter hours sleeping. However, a previous study reported that sleep had no association with prematurity [12], whereas others have found that a short duration and poor quality of sleep were both risk factors for preterm birth $[13,14]$.

A previous meta-analysis evaluated the association between sleep quality and duration with the risk of preterm birth [15], but there was significantly high heterogeneity among the included studies and no subgroup or sensitivity analysis to identify the sources of heterogeneity and test the robustness of the main findings. Since an increasing number of studies with inconsistent results have reported that preterm birth is associated with maternal sleep duration and quality, the aim of the present meta-analysis was to systematically further review the current literature regarding the impact of maternal sleep duration and quality on the risk of preterm birth.

\section{Material and methods Search strategy}

After formulating the study questions, a systematic review and meta-analysis were performed in accordance with the Preferred Reporting Items for Systematic Reviews and Meta-Analyses guidelines [16]. Based on Medical Subject Heading (MeSH) terms and key words used in recent reviews [15, 17], studies were retrieved from the PubMed and Web of Science databases with restrictions to those published in English up to September 30, 2018. The complete database search strategy is described in Table 1. Additional publications were obtained by manually searching the reference sections of primary studies and review articles.

\section{Study selection}

The titles and abstracts of the retrieved articles were screened by two independent reviewers (LW and FJ) and any disagreements were resolved by discussion. Articles that fulfilled the following criteria were eligible for the present meta-analysis: (i) participants were pregnant women who were recruited from all populations; (ii) sleep duration and quality were quantified during the term of pregnancy; (iii) preterm birth, as defined as childbirth before gestational week 37, was the primary or secondary outcome; (iv) the study used an observational design (including case-control, nested casecontrol, cross-sectional, and cohort studies); and (v) the relative risk (RR), hazard ratio (HR), or odds ratio (OR), and corresponding $95 \%$ confidence interval $(95 \% \mathrm{CI})$ or necessary data for calculation were reported. Review articles, case reports, commentaries, and conference abstracts were excluded. If data were duplicated in more than one study, the study with the largest number of cases was included.

\section{Data extraction and quality assessment}

The data from each of the included studies were extracted carefully by two independent reviewers (LW and FJ). The following information from each article was recorded in a standardized, pre-designed spreadsheet: the first author's name; publication year; country of origin; study design; number of cases/participants; source of patient recruitment; sleep assessment; pregnancy outcome categories; exposure categories (period of exposure measurement); risk estimates from the most fully adjusted model with associated 95\% CI values; and confounders adjusted for multivariate analysis.

Table 1 The search strategy of the association of sleep duration and quality with preterm birth

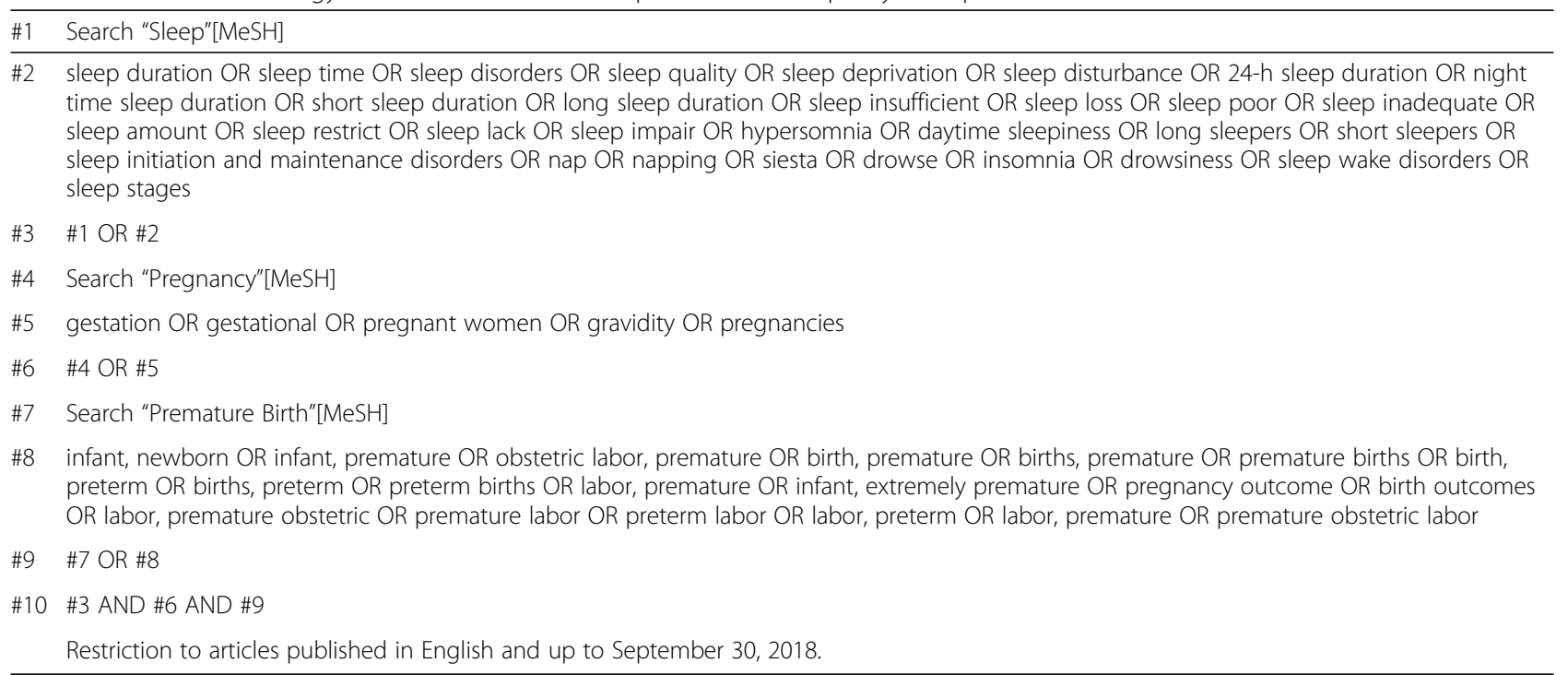


Considering that all of the included articles were observational studies, two independent reviewers (LW and FJ) used the Newcastle-Ottawa quality assessment scale for observational studies to assess the risk of bias [18]. Subsequently, the studies that achieved a full rating in at least two categories of selection, comparability, or outcome assessment were considered at a low risk of bias $[19,20]$.

\section{Statistical analysis}

For studies that separately reported results for different trimesters, but not in combination, inverse varianceweighted fixed effects meta-analysis was first used to generate a risk estimate of the overall study at full gestation before random-effects meta-analysis. For studies that failed to use the category with the shortest sleep duration as the reference, the effective count method proposed by Hamling et al. [21] was used to recalculate the risk estimates. The summary RR values and corresponding 95\% CI values of the included studies were used as measures to assess the association of sleep duration and quality with the risk of preterm birth.

Between-study heterogeneity of each meta-analysis was estimated using the $I^{2}$ statistic. $I^{2}$ values of 25,50 , and $75 \%$ were considered to represent low, moderate, and high heterogeneity, respectively [22]. Summary RR values were calculated using the DerSimonian and Laird random-effects model. The sequential exclusion strategy proposed by Patsopoulos et al. [23] was used to determine whether the overall estimates were influenced by substantial heterogeneity. Studies that accounted for the largest share of heterogeneity were sequentially and $\mathrm{cu}-$ mulatively excluded until $I^{2}$ was $<50 \%$. The RR estimates were then assessed for consistency [23]. Potential publication bias was examined with a funnel plot and the Egger's and Begg's tests $(p<0.10)$. The G*Power 3.1.9.2 statistical power analysis program was used to calculate the sample size at an alpha value of 0.05 , power of $80 \%(\beta=0.2)$, estimated RR of 1.2 , and proportion of outcome in the exposure group of 0.3 . Accordingly, the lowest sample size for a proper cohort was $n=1138$ for dichotomous outcomes. All data analyses were performed using STATA software, version 12.0 (Stata Corp, College Station, TX, USA). A probability $(p)$ value of $<$ 0.05 was considered statistically significant unless otherwise specified.

\section{Results}

\section{Characteristics of the retrieved studies}

Of the 3266 retrieved studies remaining after the removal of duplicates, 3233 (99.0\%) were excluded after screening of the titles and abstracts. After full text review of the remaining 33 (1.01\%) studies, 16 analyzed diseases and symptoms related to sleep disorders, and seven lacked OR and 95\% CI values. Among these seven studies, three suggested no association between sleep and risk of preterm birth [24-26], two indicated that short sleep was correlated with prematurity [10, 27], one showed that short and long sleep durations were associated with increased risk of preterm birth [28], and one analyzed the relationships among sleep, inflammatory cytokine levels, and preterm birth, but failed to show a direct association between sleep and preterm birth [29]. Finally, 10 published studies detailing the associations of sleep duration and quality with the risk of preterm birth were included for analysis (Fig. 1). Among these, seven and five published studies focused on sleep duration and quality, respectively.

The characteristics of the 10 studies (nine cohort studies and one case-control study) included for analysis are summarized in Tables 2 and 3. Notably, each study was published between 2011 and 2018. The number of cases in each study ranged from 12 to 479 and the number of participants/controls ranged from 116 to 1977. All of the included studies measured sleep quality and sleep duration with the use of questionnaires. The majority of studies were conducted in the USA $(n=5)$, India $(n=1)$, Greece $(n=1)$, Japan $(n=1)$, and China $(n=1)$, and were adjusted for potentially important confounders, such as body mass index $(n=4)$ and maternal age $(n=5)$. On the basis of the Newcastle-Ottawa quality assessment scale, all studies were judged to have a low risk of bias (Table 4).

\section{Sleep duration and risk of preterm birth}

The forest plots in Fig. 2 show the summarized results for the association between sleep duration and the risk of preterm birth risk in seven of the included studies, involving 1248 preterm birth cases and 5267 participants $[12,13,30-34]$. Women with the shortest sleep duration were 1.23 times more likely to have a preterm birth than those with the longest sleep duration (summarized $R R=$ $1.23 ; 95 \% \mathrm{CI}=1.01-1.50)$. Moderate heterogeneity was observed $\left(I^{2}=57.4 \%, p=0.029\right)$. Funnel plots as well as the Egger's and Begg's tests $(p=0.123$ and 0.133 , respectively) revealed no evidence of publication bias (Fig. 3).

The summarized RR values ranged from 1.41 (95\% $\mathrm{CI}=1.01-1.96, I^{2}=63.7 \%$; exclusion of Kajeepeta et al. [32]) to $1.40\left(95 \% \mathrm{CI}=0.99-1.99, I^{2}=58.9 \%\right.$; exclusion of Trivedi et al. [30]). After excluding studies that failed to adjust for potential confounders, the results were robust $(\mathrm{RR}=1.31,95 \% \mathrm{CI}=0.95-1.79)$ without heterogeneity $\left(I^{2}=54.5 \%\right)$.

\section{Sleep quality and preterm birth risk}

Five of the included studies with 156 preterm birth cases and 1230 participants examined the association between 


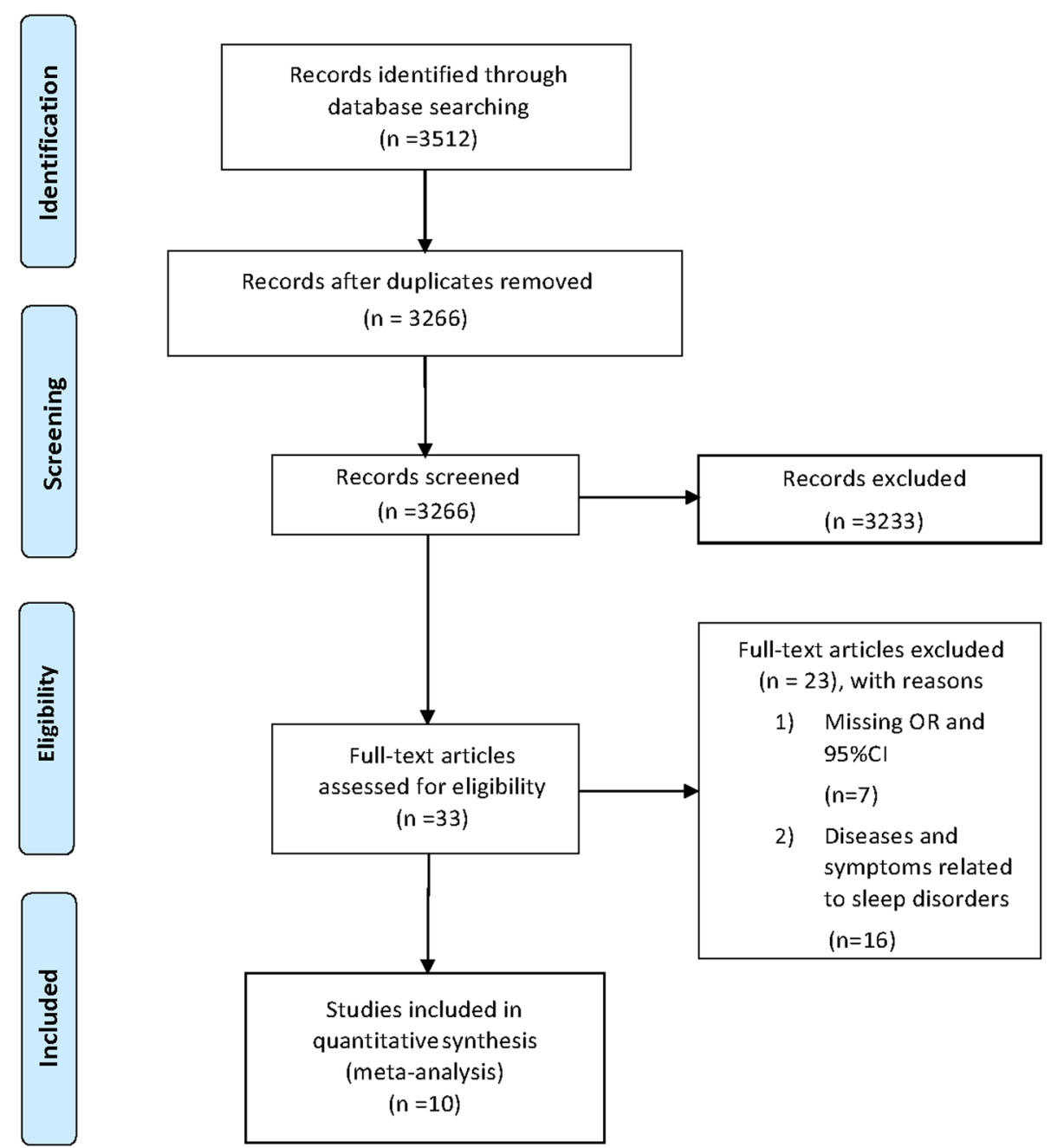

Fig. 1 Flow chart for screening of relevant literature. Selection of studies for inclusion in the present meta-analysis

sleep quality and the risk of preterm birth $[14,31,34-$ 36]. The forest plots in Fig. 4 show the summarized results for the association between sleep quality and the risk of preterm birth. Women with poor sleep quality (Pittsburgh Sleep Quality Index [PSQI] >5) were 1.54 times more likely to have a preterm birth than those with good sleep quality (summarized $\mathrm{RR}=1.54 ; 95 \%$ $\mathrm{CI}=1.18-2.01)$. High heterogeneity was observed $\left(I^{2}=\right.$ $76.7 \%, p<0.001)$. Funnel plots as well as the Egger's and Begg's tests $(p=0.221$ and 0.139 , respectively) revealed no evidence of publication bias (Fig. 5).

When the studies contributing to the largest extent of heterogeneity were sequentially excluded until $I^{2}$ was < $50 \%$ [31], the summarized RR values for preterm birth $\left(\mathrm{RR}=1.26,95 \% \mathrm{CI}=1.09-1.45, I^{2}=38.4 \%\right)$ were similar to the original estimates. Additionally, the summarized RR values ranged from $1.26\left(95 \% \mathrm{CI}=1.09-1.45, I^{2}=\right.$ $38.4 \%$; exclusion of $\mathrm{Li}$ et al. [31]) to $2.05(95 \% \mathrm{CI}=1.12-$ $3.75, I^{2}=82 \%$; exclusion of Okun et al. [33]).

\section{Discussion}

The present systematic review and meta-analysis of 10 observational studies demonstrated that short sleep duration and poor sleep quality were associated with a significantly increased risk of preterm birth. This association highlights the vital significance of pregnant women to reduce the risk of premature birth.

In regard to sleep duration and quality, the inconsistent findings of previous studies might be attributed to differences in the trimester examined and geographical location. For instance, Micheli et al. [13] conducted a cohort study $(n=1091)$, in which $23 \%$ of pregnant women reported a sleep duration of $\leq 5 \mathrm{~h}$ in the third trimester. However, Reutrakul et al. [34] reported that about $56 \%$ of pregnant women experienced sleep deprivation in the second trimester, but this study had a relatively small cohort $(n=116)$. Meanwhile, $\mathrm{Li}$ et al. [31] enrolled participants with a similar proportion of short sleepers in all trimesters and the results were 


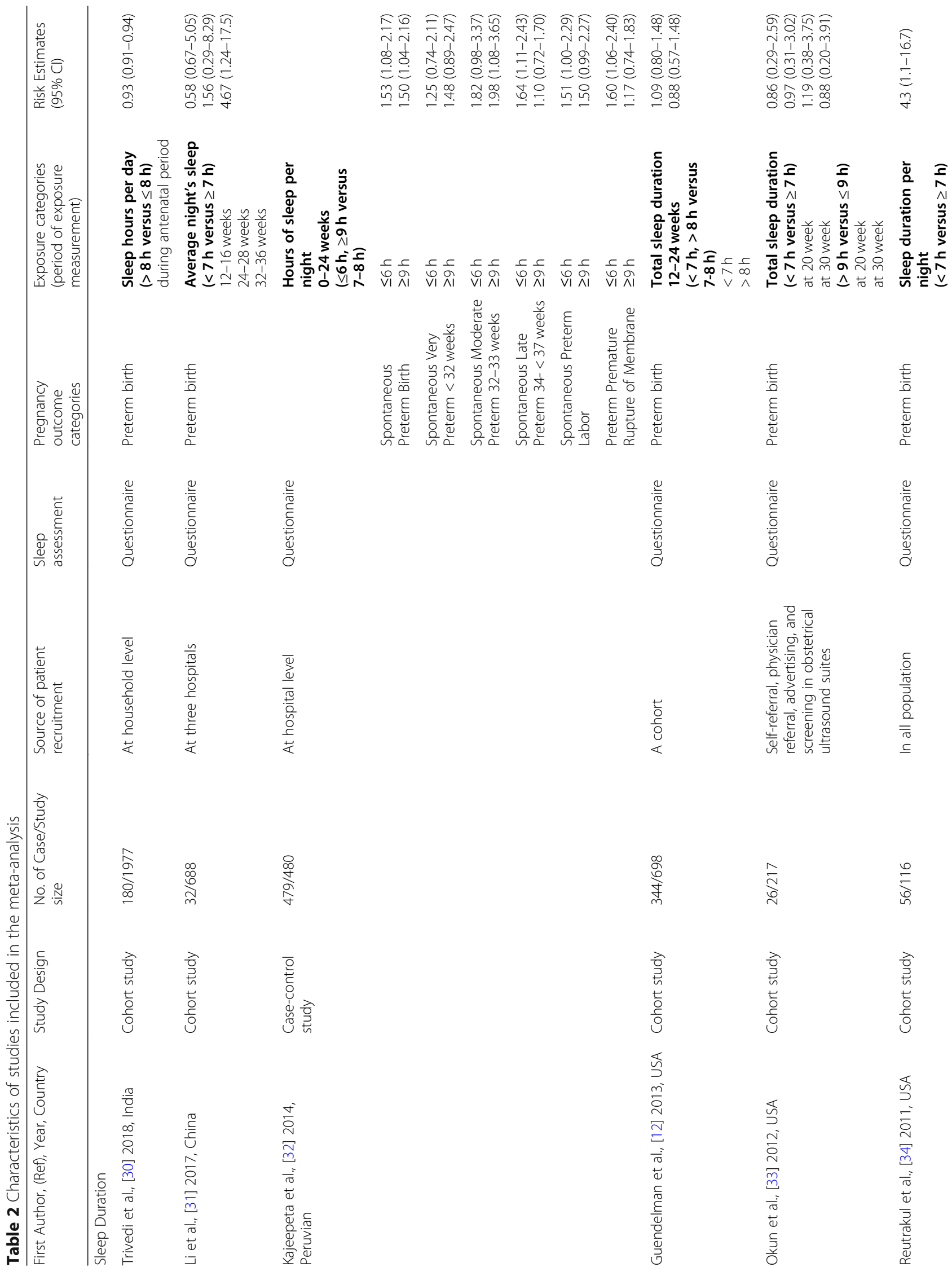




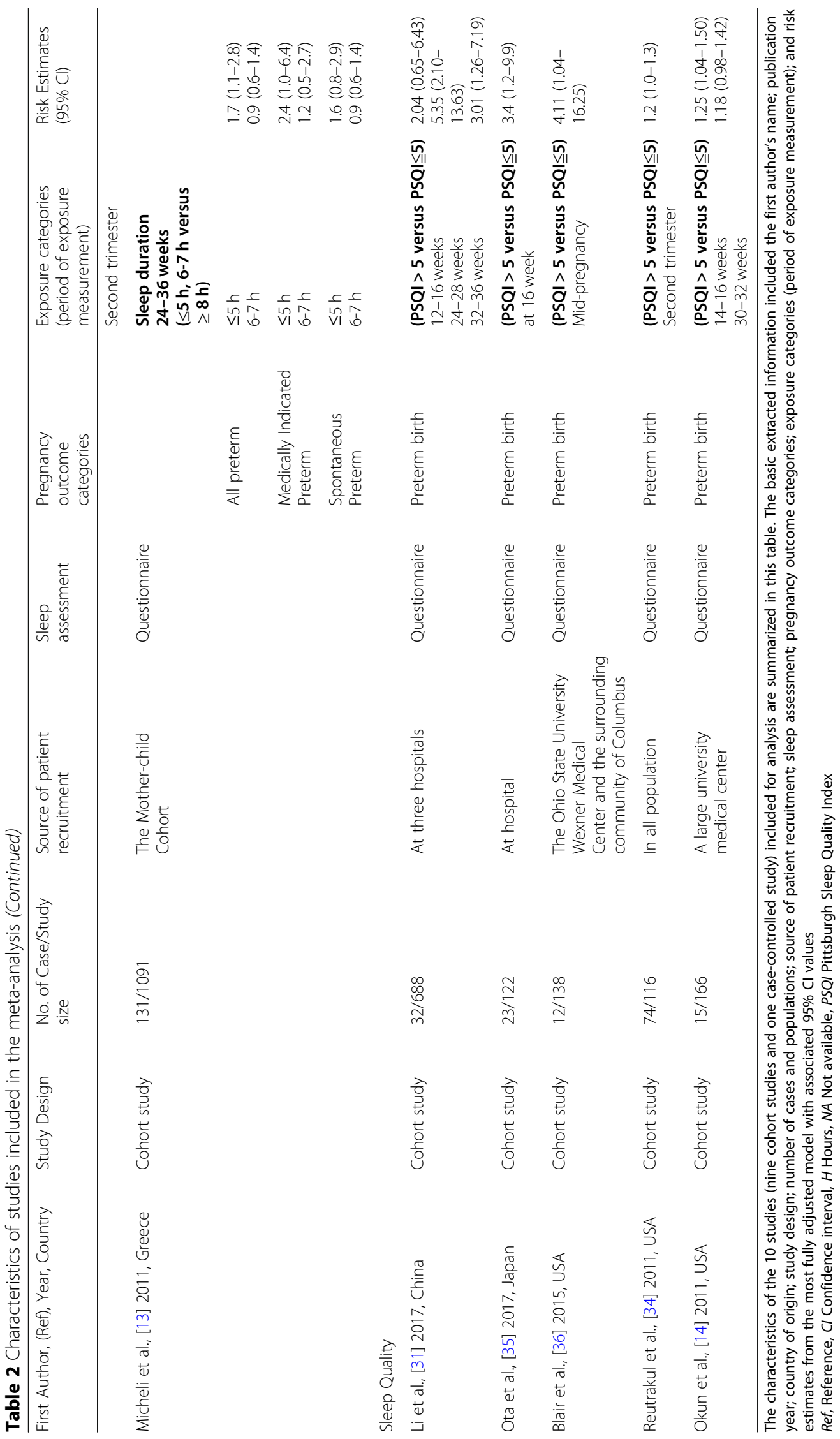


Table 3 Matched or adjustment of potential confounders of studies included in the meta-analysis

\begin{tabular}{ll}
\hline First Author, (Ref), Year, Country & Matched or adjusted factors \\
\hline Sleep Duration & \\
Trivedi et al., [30] 2018, India & Age at pregnancy, caste, religion, occupation, literacy, and number of Ante Natal Care visits \\
Li et al., [31] 2017, China & Pre-pregnancy body mass index and birth weight \\
Kajeepeta et al., [32] 2014, Peruvian & Maternal age, pre-pregnancy weight, unplanned pregnancy, and no vitamin use during pregnancy \\
Guendelman et al., [12] 2013, USA & Race and month of delivery \\
Okun et al., [33] 2012, USA & Major depressive \\
& disorder, selective serotonin reuptake inhibitors, age, employment, marital status, history of preterm birth \\
Reutrakul et al., [34] 2011, USA & Body mass index \\
Micheli et al., [13] 2011, Greece & Maternal age, education, parity, smoking during pregnancy, pre-pregnancy body mass index \\
Sleep Quality & \\
Li et al., [31] 2017, China & Pre-pregnancy body mass index and birth weight \\
Ota et al., [35] 2017, Japan & Infertility treatment, asthma, and history of alcohol use \\
Blair et al., [36] 2015, USA & Age \\
Reutrakul et al., [34] 2011, USA & Body mass index \\
Okun et al., [14] 2011, USA & Income and medical risk factors \\
\hline
\end{tabular}

The matched or adjustment of potential confounders of the studies included in the meta-analysis are summarized in this table

similar with the main findings. One study [34] reported that about $50 \%$ of pregnant women experienced sleep deprivation, but the sleep durations differed $(<7$ and $<8$ $\mathrm{h} /$ night, respectively). Furthermore, preterm birth rates vary among countries, even different regions in the same country. Also, income and education differences may affect sleep duration and preterm birth rates [37, 38]. Warland et al. [15] speculated that African Americans may exhibit heightened sensitivity to the adverse physiological sequelae of poor sleep quality. Two other studies indicated that pregnant women with clinically disturbed sleep (PSQI > 5) accounted for a similar proportion (about $60 \%$ ), despite the study being conducted in various regions within the USA $[34,36]$, while a study conducted in Japan included a lower proportion of poor sleepers at the initial examination and gestational weeks 16,24 , and 32 (27, 34, 37, and $41 \%$ of the samples, respectively) [35]. In addition, a study conducted in the USA reported that pregnant women at gestational week 14-16 accounted for $36.4 \%(n=48)$ of the sample [14].

The findings of the current study raise questions about the potential mechanisms underlying the increased risk of preterm birth due to sleep disorders. Sleep deprivation partially accounts for the proinflammatory cytokine response [39-41], immune changes [42], and greater susceptibility to infections [43]. It is well established that inflammation and infection are highly significant risk factors for preterm birth $[44,45]$. Additionally, a short sleep duration and poor sleep quality may result from stress and as a physiological stressor per se, stress "overload" and activation of the stress system may lead to prematurity through impairment of the hypothalamus-pituitary-adrenal axis and activation of the proinflammatory system [46]. On the other hand, physiological and hormonal changes also affect sleep practices. For instance, higher levels of estrogen and progesterone during pregnancy contribute to poor sleep quality and also influence the secretion of other hormones, such as cortisol and melatonin, which can increase arousal [47, 48]. Lastly, because disturbed sleep may disrupt normal remodeling of the maternal blood vessels and increased sympathetic activity, placental blood flow was decreased $[49,50]$, which may be a mechanism underlying preterm birth.

The strengths of the present meta-analysis lie in its quantitative analysis of the association of sleep duration and quality with the risk of prematurity using a large number of participants $(n=5693)$ and instances of preterm birth associated with sleep duration $(n=1248)$ and sleep quality $(n=156)$. The large sample size of this meta-analysis provide strong power for the main analyses and the conclusions derived. Furthermore, numerous sensitivity analyses showed that the main findings were robust. Of note, quality assessment showed that all of the included studies were at a low risk of bias.

Findings from the present meta-analysis should be interpreted in light of several limitations. First, the present meta-analysis was prone to inherent recall and selection bias due to the inclusion of original observational studies. Although case-control studies are more susceptible to bias than cohort studies, the results were robust after exclusion of the only case-control study from the analyses. Furthermore, the PSQI is an important clinical and research tool to gauge sleep quality [51]. However, the PSQI includes sleep duration, thus short sleep duration was included as an outcome of "sleep quality." Consistently, the pooled effect size for poor sleep quality $(R R=1.54)$ was similar to that for a shorter 


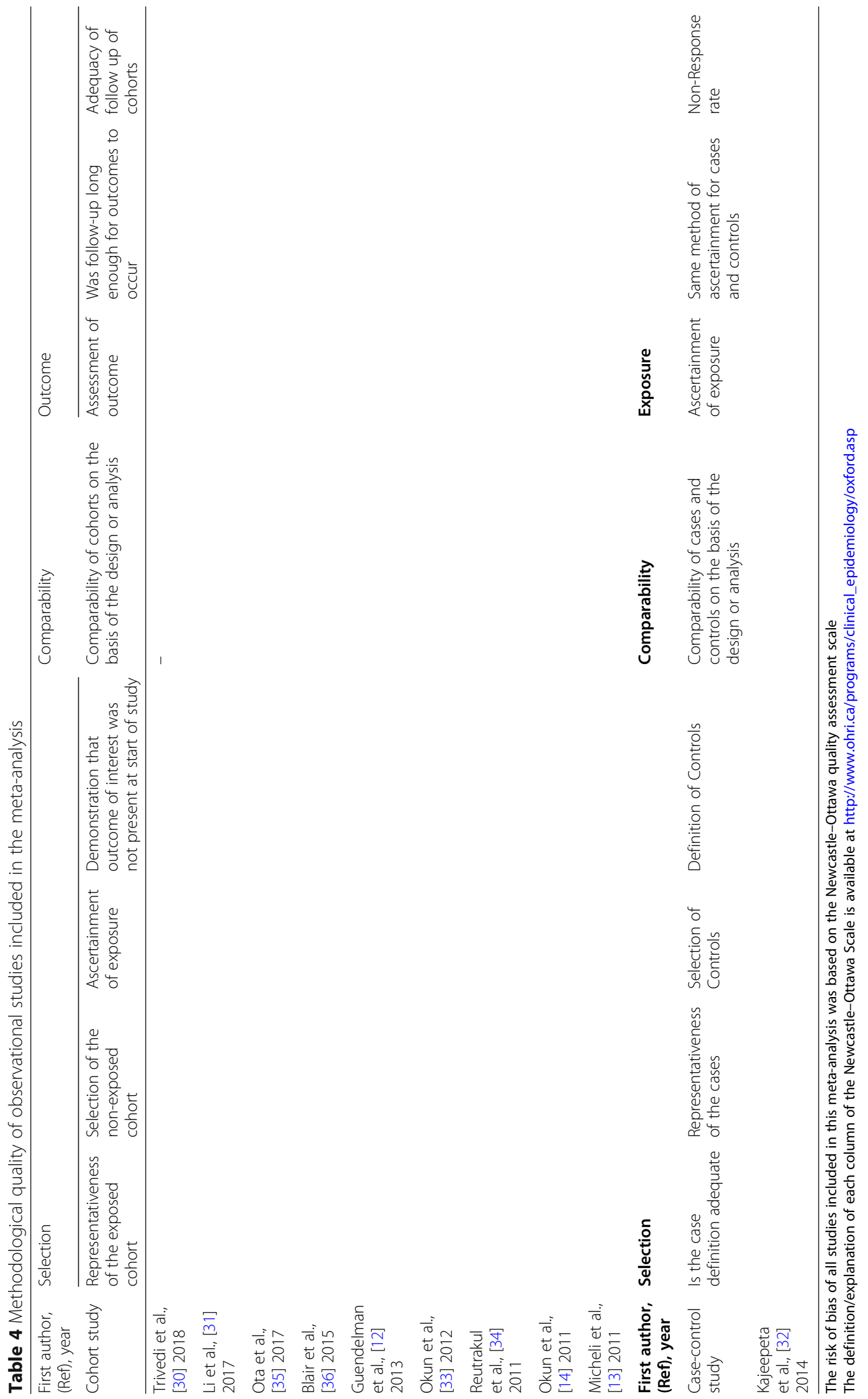




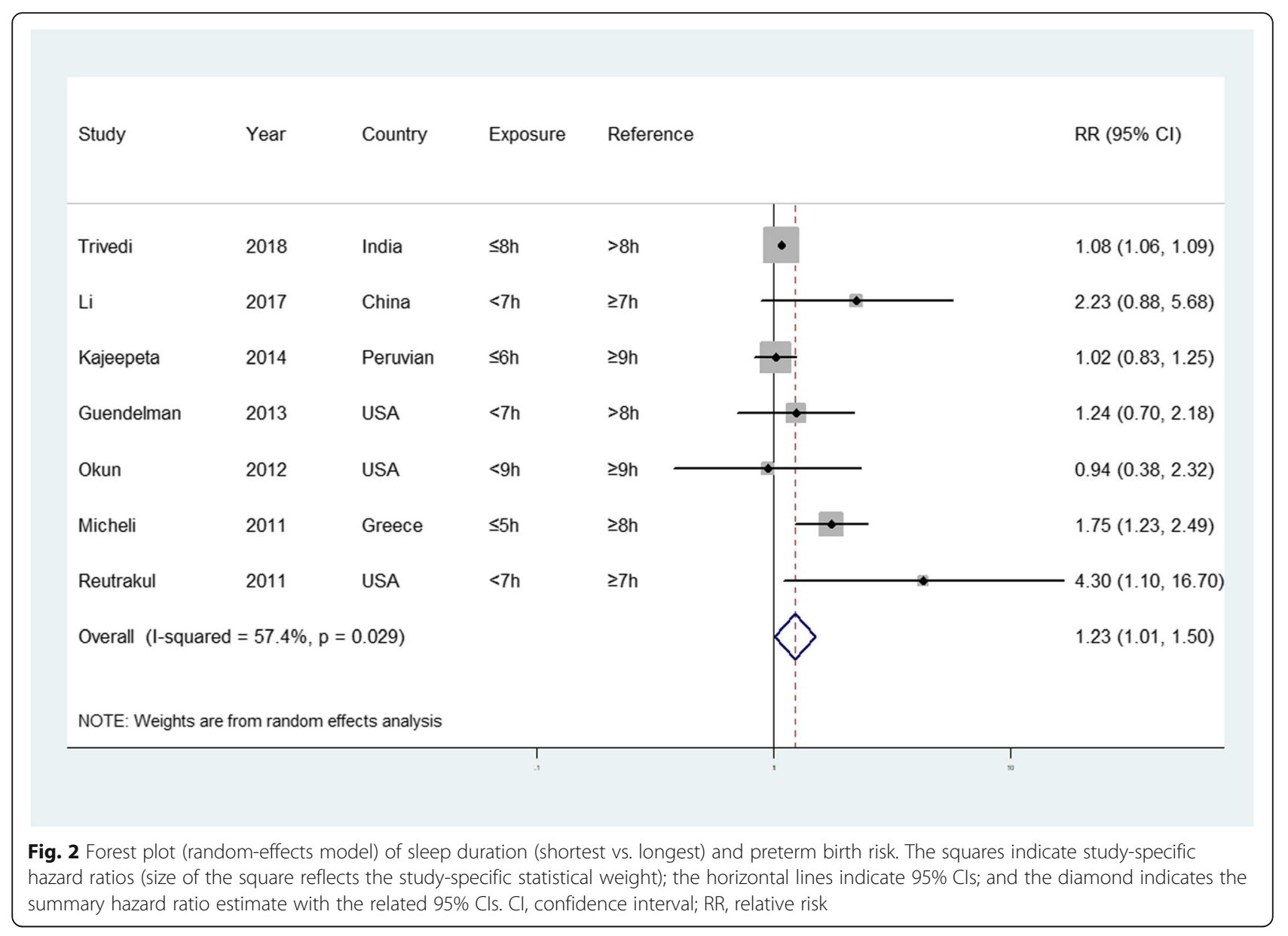

Fig. 2 Forest plot (random-effects model) of sleep duration (shortest vs. longest) and preterm birth risk. The squares indicate study-specific hazard ratios (size of the square reflects the study-specific statistical weight); the horizontal lines indicate 95\% Cls; and the diamond indicates the summary hazard ratio estimate with the related $95 \% \mathrm{Cls}$. Cl, confidence interval; RR, relative risk

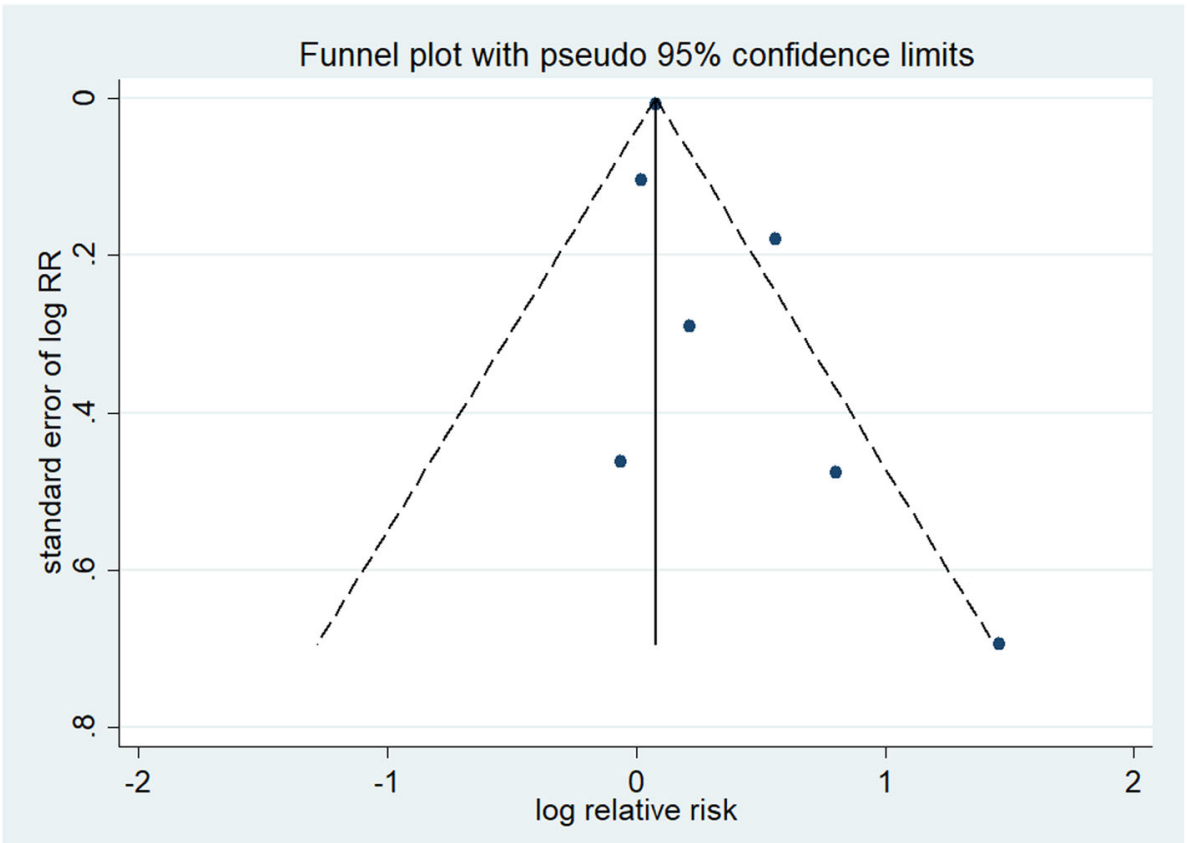

Fig. 3 Funnel plot corresponding to the random-effects meta-analysis of the relationship between sleep duration and preterm birth risk 


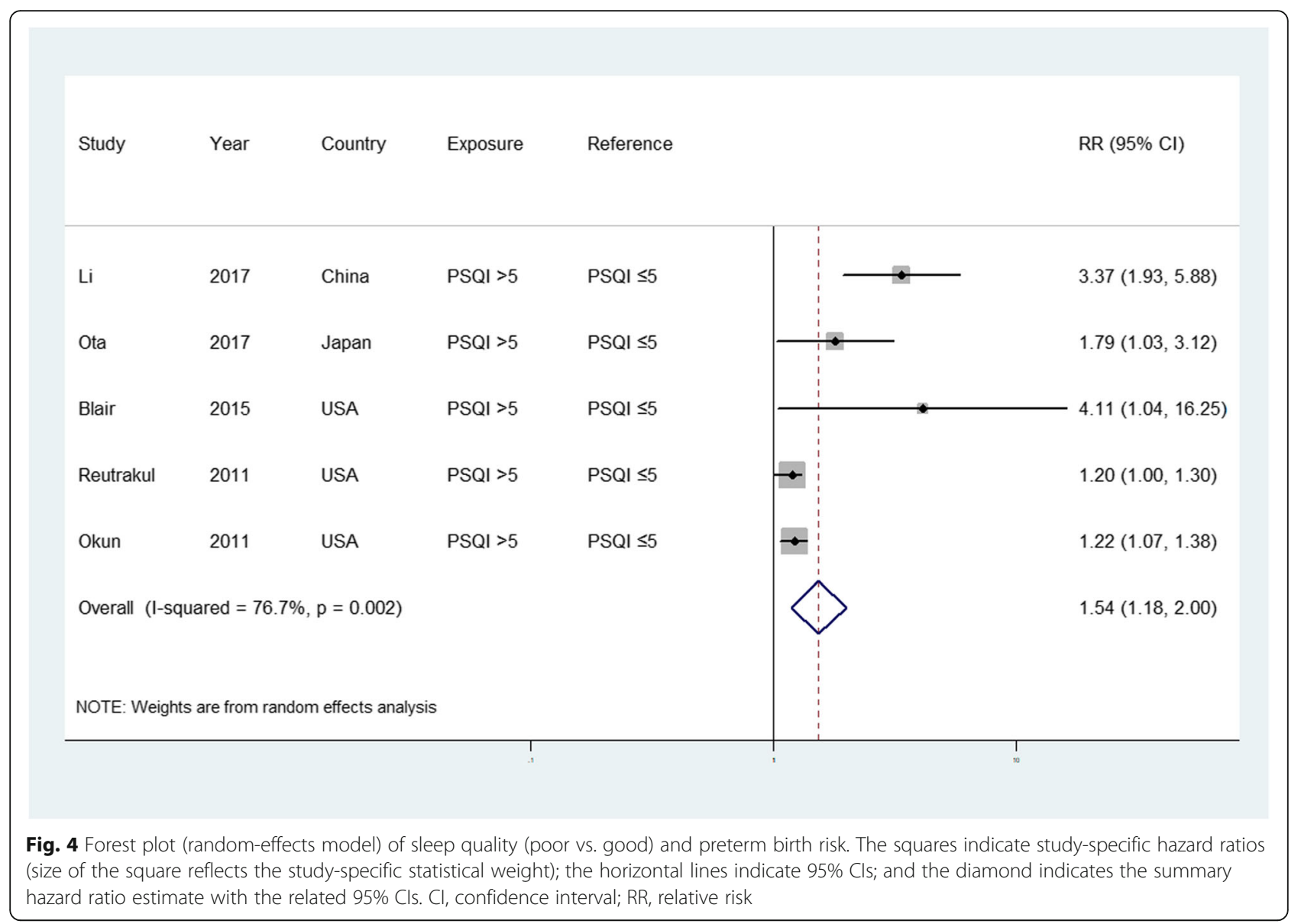
(size of the square reflects the study-specific statistical weight); the horizontal lines indicate $95 \% \mathrm{Cls}$; and the diamond indicates the summary hazard ratio estimate with the related $95 \% \mathrm{Cls}$. $\mathrm{Cl}$, confidence interval; $\mathrm{RR}$, relative risk

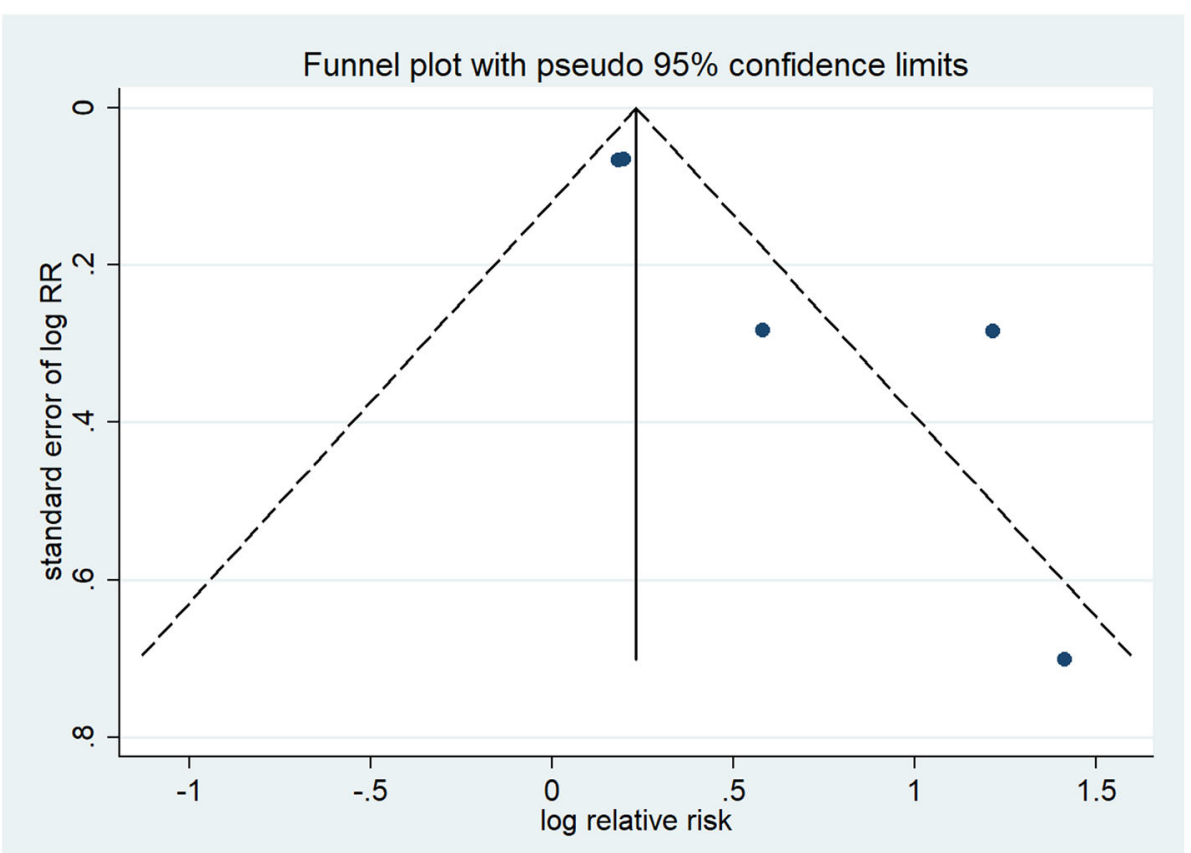

Fig. 5 Funnel plot corresponding to the random-effects meta-analysis of the relationship between sleep quality and preterm birth risk 
duration $(R R=1.32)$, as determined by the metaanalysis. Moreover, in consideration of the variation of the study populations, geographical location likely contributed to the heterogeneity of effect estimates. Furthermore, since all of the included studies measured sleep quality and sleep duration with the use of questionnaires, self-reported sleep quality and duration are not always perfectly aligned with objective sleep quality and duration. Third, because the pooled effect estimates were mostly derived from observational studies, susceptibility to confounding factors remain a concern.

Some common chronic diseases, as mediators between short sleep duration and preterm birth, such as diabetes $[34,52]$, hypertension [53, 54], and obesity [55], have been correlated with prematurity. Of note, self-reported sleep disturbances are predictive of the incidence of major depression and strongly precede a series of symptoms of depression $[56,57]$. The association between depression syndrome and the risk of preterm delivery has been reported [58]. Thus, early intervention to prevent poor sleep quality and a short sleep duration, which may be indicators of early depression, can reduce the risk of preterm birth. However, the observational studies included in this meta-analysis were restricted by the lack of controls for these potentially relevant confounders. Hence, further studies are warranted with better designs to take these confounders or mediators into account or fully adjust for these confounders in order to better rule out the potential effects of residual confounding. Fourth, as the comparison of sleep duration differed considerably among the included studies, dose-response analysis was not conducted. Notably, several of the included studies suggested a potential U-shaped association between sleep duration and preterm birth. Additionally, one of the included studies suggested a potential nonlinear (U-shaped) association between sleep duration and preterm birth [32]. However, since a limited number of the included studies met the criteria of linear/non-linear dose-response analysis, such analysis was not conducted in the present study. Also, although seven studies were excluded due to the lack of risk estimates for the association between sleep quantity/quality and preterm birth [10, 24-29], findings of three of these studies support the main findings of the present meta-analysis [10, $27,28]$. Of note, although the power of the main analysis suggested that the statistical power of this meta-analysis was greater than $80 \%$ to identify sleep duration/quality for preterm birth with minimum OR values of 1.20 (risk factor for sleep duration) and 1.5 (risk factor for sleep quality), limited sample sizes restricted subgroup analyses stratified by study characteristics and potential confounders. Therefore, on the basis of these limitations, priority should be given to large, adequately powered, cohort studies using standard definitions of maternal sleep duration and quality with effective data analyses. Furthermore, more comparison groups in the primary studies are needed to evaluate the possible non-linear aforementioned association.

\section{Conclusion}

In summary, the results of this meta-analysis provide vital insights into the association of short sleep duration and poor sleep quality with a significantly increased risk of preterm birth. These findings may help researchers to identify women at risk of pre-term birth early during pregnancy to provide targeted interventions.

\section{Abbreviations}

95\% Cl: 95\% confidence interval; HR: Hazard ratio; NA: Not available; OR: Odds ratio; PSQI: Pittsburgh sleep quality index.; Ref: Reference; RR: Relative risk

\section{Acknowledgments}

Not applicable.

\section{Author contributions}

Conception of the study: LW, FJ; data collection: LW; data analysis and interpretation: LW, FJ; drafting the article: LW, FJ; critical revision of the article: FJ; final approval of the version to the published: LW, FJ.

\section{Funding}

This work was supported by a grant from the Natural Science Foundation of Liaoning Providence (grant no. 2019JH3/10300448 to Feng Jin).

\section{Availability of data and materials}

All data generated or analyzed in this study are included in the referenced articles.

Ethics approval and consent to participate Not applicable.

Consent for publication

Not applicable.

\section{Competing interests}

The authors have no competing interests to declare.

Received: 8 July 2019 Accepted: 14 February 2020

Published online: 24 February 2020

\section{References}

1. Liu L, Oza S, Hogan D, Perin J, Rudan I, Lawn JE, Cousens S, Mathers C, Black RE. Global, regional, and national causes of child mortality in 2000-13, with projections to inform post-2015 priorities: an updated systematic analysis. Lancet (London, England). 2015;385(9966):430-40.

2. Born Too Soon:The Global Action Report on Preterm Birth [http://www.who. int/pmnch/media/news/2012/preterm_birth_report/en/index3.html]. Accessed 2 Jan 2020.

3. Khashu M, Narayanan M, Bhargava S, Osiovich H. Perinatal outcomes associated with preterm birth at 33 to 36 Weeks' gestation: a populationbased cohort study. Pediatrics. 2009;123(1):109-13.

4. Allen MC. Neurodevelopmental outcomes of preterm infants. Curr Opin Neurol. 2008;21(2):123-8

5. Children:Reducing Mortality [http://www.who.int/mediacentre/factsheets/fs 1 78/en/]. Accessed 11 Dec 2019.

6. Vogel JP, Chawanpaiboon S, Moller AB, Watananirun K, Bonet M, Lumbiganon P. The global epidemiology of preterm birth. Best Pract Res Clin Obstet Gynaecol. 2018:52:3-12.

7. Baer RJ, McLemore MR, Adler N, Oltman SP, Chambers BD, Kuppermann M, Pantell MS, Rogers EE, Ryckman KK, Sirota M, et al. Pre-pregnancy or firsttrimester risk scoring to identify women at high risk of preterm birth. Eur J Obstet Gynecol Reprod Biol. 2018;231:235-40. 
8. Reid KJ, Facco FL, Grobman WA, Parker CB, Herbas M, Hunter S, Silver RM, Basner RC, Saade GR, Pien GW, et al. Sleep During Pregnancy: The nuMoM2b Pregnancy and Sleep Duration and Continuity Study. Sleep. 2017; 40(5).

9. Maxmen A. Insomnia linked to premature birth in study of 3 million mothers. Nature. 2017:548(7666):145.

10. Meharaban Z, Yahya S, Sadegniiat K. Restless Legs Syndrome During Pregnancy and Preterm Birth in Women Referred to Health Centers of Ardabil. Iran Red Crescent Med J. 2015:17(12).

11. Suzuki K, Ohida T, Sone T, Takemura S, Yokoyama E, Miyake T, Harano S, Nozaki N, Motojima S, Suga M, et al. an epidemiological study of sleep problems among the Japanese pregnant women. Nihon Koshu Eisei Zasshi. 2003;50(6):526-39.

12. Guendelman S, Pearl M, Kosa JL, Graham S, Abrams B, Kharrazi M. Association between preterm delivery and pre-pregnancy body mass (BMI), exercise and sleep during pregnancy among working women in Southern California. Matern Child Health J. 2013;17(4):723-31.

13. Micheli K, Komninos I, Bagkeris E, Roumeliotaki T, Koutis A, Kogevinas M, Chatzi L. Sleep patterns in late pregnancy and risk of preterm birth and fetal growth restriction. Epidemiology (Cambridge, Mass). 2011;22(5):738-44.

14. Okun ML, Schetter CD, Glynn LM. Poor sleep quality is associated with preterm birth. Sleep. 2011;34(11):1493-8.

15. Warland J, Dorrian J, Morrison JL, O'Brien LM. Maternal sleep during pregnancy and poor fetal outcomes: a scoping review of the literature with meta-analysis. Sleep Med Rev. 2018;41:197-219.

16. Knobloch K, Yoon U, Vogt PM. Preferred reporting items for systematic reviews and meta-analyses (PRISMA) statement and publication bias. J Cranio-Maxillo-Facial Surg : Official Publication Eur Assoc Cranio-MaxilloFacial Surg. 2011;39(2):91-2.

17. Hashmi AM, Bhatia SK, Bhatia SK, Khawaja IS. Insomnia during pregnancy: diagnosis and rational interventions. Pak J Med Sci. 2016;32(4):1030-7.

18. The Newcastle-Ottawa Scale (NOS) for assessing the quality of nonrandomised studies in meta-analyses [http://www.ohri.ca/programs/ clinical_epidemiological/oxford.asp]. Accessed 19 Nov 2019.

19. Gao SY, Wu QJ, Sun C, Zhang TN, Shen ZQ, Liu CX, Gong TT, Xu X, Ji C, Huang $\mathrm{DH}$, et al. Selective serotonin reuptake inhibitor use during early pregnancy and congenital malformations: a systematic review and metaanalysis of cohort studies of more than 9 million births. BMC Med. 2018; 16(1):205

20. Odutayo A, Wong CX, Hsiao AJ, Hopewell S, Altman DG, Emdin CA. Atrial fibrillation and risks of cardiovascular disease, renal disease, and death: systematic review and meta-analysis. BMJ (Clinical research ed). 2016;354: i4482.

21. Hamling J, Lee $P$, Weitkunat $R$, Ambuhl M. Facilitating meta-analyses by deriving relative effect and precision estimates for alternative comparisons from a set of estimates presented by exposure level or disease category. Stat Med. 2008;27(7):954-70

22. Chen LH, Wang YF, Xu QH, Chen SS. Omega-3 fatty acids as a treatment for non-alcoholic fatty liver disease in children: A systematic review and metaanalysis of randomized controlled trials. Clinical Nutrition (Edinburgh, Scotland). 2018;37(2):516-21.

23. Patsopoulos NA, Evangelou E, loannidis JP. Sensitivity of between-study heterogeneity in meta-analysis: proposed metrics and empirical evaluation. Int J Epidemiol. 2008;37(5):1148-57.

24. Hernandez-Diaz S, Boeke CE, Romans AT, Young B, Margulis AV, McElrath TF, Ecker JL, Bateman BT. Triggers of spontaneous preterm delivery - why today? Paediatr Perinat Epidemiol. 2014;28(2):79-87.

25. Owusu JT, Anderson FJ, Coleman J, Oppong S, Seffah JD, Aikins A, O'Brien LM. Association of maternal sleep practices with pre-eclampsia, low birth weight, and stillbirth among Ghanaian women. Int J Gynaecol Obstet: Official Organ Int Fed Gynaecol Obstet. 2013;121(3):261-5.

26. Strange LB, Parker KP, Moore ML, Strickland OL, Bliwise DL. Disturbed sleep and preterm birth: a potential relationship? Clin Exp Obstet Gynecol. 2009; 36(3):166-8.

27. Dolatian M, Mehraban Z, Sadeghniat K. The effect of impaired sleep on preterm labour. West Indian Med J. 2014;63(1):62-7.

28. Plancoulaine S, Flori S, Bat-Pitault F, Patural H, Lin J-S, Franco P. Sleep trajectories among pregnant women and the impact on outcomes: a population-based cohort study. Matern Child Health J. 2017;21(5):1139-46.

29. Okun ML, Luther JF, Wisniewski SR, Wisner KL. Disturbed sleep and inflammatory cytokines in depressed and nondepressed pregnant women: an exploratory analysis of pregnancy outcomes. Psychosom Med. 2013; 75(7):670-81.

30. Trivedi P, Saxena D, Puwar T, Savaliya S, Ganguly P. A cohort study on risk factors for preterm births in rural Gujarat. Indian J Public Health. 2018;62(2): $111-6$.

31. Li R, Zhang J, Zhou R, Liu J, Dai Z, Liu D, Wang Y, Zhang H, Li Y, Zeng G. Sleep disturbances during pregnancy are associated with cesarean delivery and preterm birth. J Maternal-Fetal Neonatal Med : Official J Eur Assoc Perinat Med, Fed Asia Oceania Perinat Soc, Int Soc Perinat Obstet. 2017; 30(6):733-8.

32. Kajeepeta S, Sanchez SE, Gelaye B, Qiu C, Barrios W, Enquobahrie DA, Williams MA. Sleep duration, vital exhaustion, and odds of spontaneous preterm birth: a case-control study. BMC pregnancy and childbirth. 2014;14:337.

33. Okun ML, Luther JF, Wisniewski SR, Sit D, Prairie BA, Wisner KL. Disturbed sleep, a novel risk factor for preterm birth? J Women's Health (2002). 2012; 21(1):54-60

34. Reutrakul S, Zaidi N, Wroblewski K, Kay HH, Ismail M, Ehrmann DA, Van Cauter E. Sleep disturbances and their relationship to glucose tolerance in pregnancy. Diabetes Care. 2011;34(11):2454-7.

35. Ota H, Hasegawa J, Sekizawa A. Effect of sleep disorders on threatened premature delivery. J Perinat Med. 2017;45(1):57-61.

36. Blair LM, Porter K, Leblebicioglu B, Christian LM. Poor sleep quality and associated inflammation predict preterm birth: heightened risk among African Americans. Sleep. 2015;38(8):1259-67.

37. Begum F, Buckshe K, Pande JN. Risk factors associated with preterm labour. Bangladesh Med Res Counc Bull. 2003;29(2):59-66.

38. Rao $C R$, de Ruiter $L E$, Bhat $P$, Kamath $V$, Kamath $A$, Bhat $V$. A case-control study on risk factors for preterm deliveries in a secondary care hospital, southern India. ISRN Obstet Gynecol. 2014;2014:935982.

39. Irwin MR, Wang M, Campomayor CO, Collado-Hidalgo A, Cole S. Sleep deprivation and activation of morning levels of cellular and genomic markers of inflammation. Arch Intern Med. 2006;166(16):1756-62.

40. Okun ML, Coussons-Read ME. Sleep disruption during pregnancy: how does it influence serum cytokines? J Reprod Immunol. 2007;73(2):158-65.

41. Holingue C, Owusu JT, Feder KA, Spira AP. Sleep duration and C-reactive protein: associations among pregnant and non-pregnant women. J Reprod Immunol. 2018;128:9-15.

42. Irwin M. Effects of sleep and sleep loss on immunity and cytokines. Brain Behav Immun. 2002;16(5):503-12.

43. Ibarra-Coronado EG, Pantaleon-Martinez AM, Velazquez-Moctezuma J, Prospero-Garcia O, Mendez-Diaz M, Perez-Tapia M, Pavon L, Morales-Montor $J$. The bidirectional relationship between sleep and immunity against infections. J Immunol Res. 2015;2015:678164.

44. Cappelletti M, Della Bella S, Ferrazzi E, Mavilio D, Divanovic S. Inflammation and preterm birth. J Leukoc Biol. 2016;99(1):67-78.

45. Wei SQ, Fraser W, Luo ZC. Inflammatory cytokines and spontaneous preterm birth in asymptomatic women: a systematic review. Obstet Gynecol. 2010;116(2 Pt 1):393-401.

46. Palagini L, Gemignani A, Banti S, Manconi M, Mauri M, Riemann D. Chronic sleep loss during pregnancy as a determinant of stress: impact on pregnancy outcome. Sleep Med. 2014;15(8):853-9.

47. Miller EH. Women and insomnia. Clin Cornerstone. 2004;6(Suppl 1B):S8-18.

48. Teran-Perez G, Arana-Lechuga Y, Esqueda-Leon E, Santana-Miranda R, RojasZamorano JA, Velazquez Moctezuma J. Steroid hormones and sleep regulation. Mini Revi Med Chem. 2012;12(11):1040-8.

49. Izci-Balserak B, Pien GW. Sleep-disordered breathing and pregnancy: potential mechanisms and evidence for maternal and fetal morbidity. Curr Opin Pulm Med. 2010;16(6):574-82.

50. Okun ML, Roberts JM, Marsland AL, Hall M. How disturbed sleep may be a risk factor for adverse pregnancy outcomes. Obstet Gynecol Surv. 2009; 64(4):273-80.

51. Sedov ID, Cameron EE, Madigan S, Tomfohr-Madsen LM. Sleep quality during pregnancy: a meta-analysis. Sleep Med Rev. 2018;38:168-76.

52. Gangwisch JE, Heymsfield SB, Boden-Albala B, Buijs RM, Kreier F, Pickering TG, Rundle AG, Zammit GK, Malaspina D. Sleep duration as a risk factor for diabetes incidence in a large U.S. sample. Sleep. 2007;30(12):1667-73.

53. Vgontzas AN, Liao D, Bixler EO, Chrousos GP, Vela-Bueno A. Insomnia with objective short sleep duration is associated with a high risk for hypertension. Sleep. 2009;32(4):491-7.

54. Williams MA, Miller RS, Qiu C, Cripe SM, Gelaye B, Enquobahrie D. Associations of early pregnancy sleep duration with trimester-specific 
blood pressures and hypertensive disorders in pregnancy. Sleep. 2010; 33(10):1363-71.

55. Kohatsu ND, Tsai R, Young T, Vangilder R, Burmeister LF, Stromquist AM, Merchant JA. Sleep duration and body mass index in a rural population. Arch Intern Med. 2006;166(16):1701-5.

56. Perlis ML, Giles DE, Buysse DJ, Tu X, Kupfer DJ. Self-reported sleep disturbance as a prodromal symptom in recurrent depression. J Affect Disord. 1997;42(2-3):209-12.

57. Roberts RE, Duong HT. The prospective association between sleep deprivation and depression among adolescents. Sleep. 2014;37(2):239-44.

58. Li D, Liu L, Odouli R. Presence of depressive symptoms during early pregnancy and the risk of preterm delivery: a prospective co<phort study. Hum Reprod (Oxford, England). 2009;24(1):146-53.

\section{Publisher's Note}

Springer Nature remains neutral with regard to jurisdictional claims in published maps and institutional affiliations.

Ready to submit your research? Choose BMC and benefit from:

- fast, convenient online submission

- thorough peer review by experienced researchers in your field

- rapid publication on acceptance

- support for research data, including large and complex data types

- gold Open Access which fosters wider collaboration and increased citations

- maximum visibility for your research: over $100 \mathrm{M}$ website views per year

At BMC, research is always in progress.

Learn more biomedcentral.com/submissions 